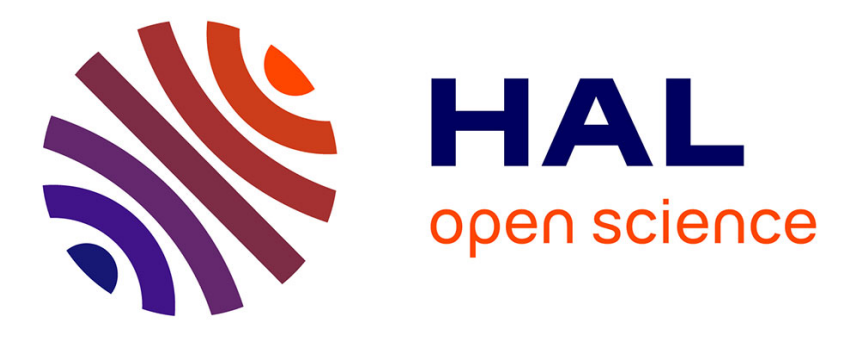

\title{
Scaling property for fragmentation processes related to avalanches
}

\author{
Lucian Beznea, Madalina Deaconu, Oana Lupaşcu-Stamate
}

\section{To cite this version:}

Lucian Beznea, Madalina Deaconu, Oana Lupaşcu-Stamate. Scaling property for fragmentation processes related to avalanches. Applications of Mathematics and Informatics in Natural Sciences and Engineering, Applications of Mathematics and Informatics in Natural Sciences and Engineering AMINSE 2019, Tbilisi, Georgia, September 23-26 (334), Springer, 2021, Springer Proceedings in Mathematics \& Statistics, 10.1007/978-3-030-56356-1_3 . hal-02942710

\section{HAL Id: hal-02942710 https://hal.science/hal-02942710}

Submitted on 18 Sep 2020

HAL is a multi-disciplinary open access archive for the deposit and dissemination of scientific research documents, whether they are published or not. The documents may come from teaching and research institutions in France or abroad, or from public or private research centers.
L'archive ouverte pluridisciplinaire HAL, est destinée au dépôt et à la diffusion de documents scientifiques de niveau recherche, publiés ou non, émanant des établissements d'enseignement et de recherche français ou étrangers, des laboratoires publics ou privés. 


\title{
Scaling property for fragmentation processes related to avalanches
}

\author{
Lucian Beznea, Madalina Deaconu, and Oana Lupaşcu-Stamate
}

\begin{abstract}
We emphasize a scaling property for the continuous time fragmentation processes related to a stochastic model for the fragmentation phase of an avalanche. We present numerical results that confirm the validity of the scaling property for our model, based on the appropriate stochastic differential equation of fragmentation and on a fractal property of the solution.
\end{abstract}

Keywords scaling, fragmentation stochastic differential equation, jump process, Monte Carlo method

Mathematics Subject Classification 39A13, 65C30, 60J45, 65C05, 60J35

\section{Introduction}

In the papers [1], [2], and [3] we studied binary fragmentation processes (and associated non-local branching processes, cf. [4]) of an infinite particles system, including a numerical approach for the time evolution of the fragmentation phase of an avalanche. A fractal property was emphasized for the process related to the avalanches.

Lucian Beznea

Simion Stoilow Institute of Mathematics of the Romanian Academy, P.O. Box 1-764, RO-014700

Bucharest, Romania, University of Bucharest, Faculty of Mathematics and Computer Science, and Centre Francophone en Mathématique de Bucarest, e-mail: lucian.beznea@imar.ro

Madalina Deaconu

Université de Lorraine, CNRS, Inria, IECL, F-54000 Nancy, France,

e-mail: Madalina.Deaconu@inria.fr

Oana Lupaşcu-Stamate

Gheorghe Mihoc-Caius Iacob Institute of Mathematical Statistics and Applied Mathematics of the Romanian Academy, Calea 13 Septembrie 13, RO-050711 Bucharest, Romania, e-mail: oana.lupascu_stamate@yahoo.com 
In this paper we prove that a scaling property holds for the above mentioned process and we present numerical results that confirm the validity of this property.

The study of the scaling property is closely related to the study of the selfsimilarity property. In this direction some results were recently obtained by using deterministic approaches. In particular in [5] this study is performed by studying the asymptotic behaviour of the first eigenvalue, as it represents the asymptotic growth of the solution. In the presence of a transport term it is shown that this behaviour depends on wether transport dominates fragmentation or not. This equation has some applications in biology and medicine. Previously in [7] the self-similarity property was used for the coagulation-fragmentation equation to obtain the existence of a stationary solution for any given mass. In [10] the fragmentation is used to model cell-division and the authors prove the existence of a stable steady distribution.

The paper is organised as follows.

In Sect. 2 we present a general result, characterizing (in Theorem 1) the scaling property of Markov process in terms of the transition function, the associated resolvent of kernels, and of the generator. It is pointed out also the case of a pure jump process.

In Sect. 3 it is given the main application, by proving (in Corollary 1) that the weak solution of the stochastic differential equation of fragmentation for avalanches has a scaling property. As it was already mentioned, it is a second specific property emphasized for this SDE of fragmentation, the first one being the fractal property proved in [2], and for the reader convenience we presented it at the end of the section.

Finally, in Sect. 4 we discuss the numerical results, obtained by Monte Carlo simulation, that confirm the validity of the scaling property we proved.

\section{Scaling property for jump processes}

Let $E$ be Lusin topological space (i.e., $E$ is homeomorphic to a Borel subset of a compact metric space) with Borel $\sigma$-algebra $\mathscr{B}(E)$. We denote by $p \mathscr{B}(E)$ (resp. $b \mathscr{B}(E)$ ) the set of all positive Borel measurable functions on $E$ (resp. the set of all bounded real-valued Borel measurable functions on $E$ ).

Let $X=\left(\Omega, \mathscr{F}, \mathscr{F}_{t}, X_{t}, \theta_{t}, \mathbb{P}^{x}, \zeta\right)$ be the right Markov process on $E$ having $\left(P_{t}\right)_{t \geqslant 0}$ as transition function, $P_{t} f(x)=\mathbb{E}^{x}\left(f\left(X_{t}\right), t<\zeta\right), t \geqslant 0, f \in p \mathscr{B}(E)$. Let further $\left(U_{\alpha}\right)_{\alpha>0}$ be the associated sub-Markovian resolvent of kernels, $U_{\alpha} f:=$ $\int_{0}^{\infty} e^{-\alpha t} P_{t} f \mathrm{~d} t$.

We consider the generator $(L, \mathscr{D}(L))$ of $X$ as follows; cf. [6] pag. 55, and [9]. Let

$$
\mathscr{B}_{o}:=\left\{f \in b \mathscr{B}(E): \lim _{t \searrow 0} P_{t} f=f \text { pointwise on } E\right\}
$$

and $\mathscr{D}(L)$ be the set of all $f \in \mathscr{B}_{o}$ such that $\left(\frac{P_{t} f(x)-f(x)}{t}\right)_{t, x}$ is bounded for $x \in E$ and $t$ in a neighbourhood of zero, there exists $\lim _{t \searrow 0} \frac{P_{t} f-f}{t}$ pointwise and the above 
limit is an element of $\mathscr{B}_{o}$. Define the linear operator $L: \mathscr{D}(L) \longrightarrow b \mathscr{B}(E)$ as

$$
L f(x):=\lim _{t \searrow 0} \frac{P_{t} f(x)-f(x)}{t}, f \in \mathscr{D}(L), x \in E .
$$

The operator $(L, \mathscr{D}(L))$ is called the weak generator of $X$. Recall that $\mathscr{D}(L)=$ $U_{\alpha}\left(\mathscr{B}_{o}\right)$ for all $\alpha>0$, and if $f=U_{\alpha} g$, with $g \in \mathscr{B}_{o}$, then $(\alpha-L) f=g$.

We present now the classical construction of a jump process (see, e.g., [8], page 163), as we need it for the fragmentations processes related to avalanches.

Let $N$ be a bounded kernel on $E$ and denote by $\lambda(x)$ the total mass of the measure $N_{x}, x \in E, \lambda(x):=N 1(x) \in E$. We set

$$
\lambda_{o}:=\|N 1\|_{\infty} \text { and } N^{\prime}:=\frac{1}{\lambda_{o}} N+\left(1-\frac{\lambda}{\lambda_{o}}\right) I,
$$

and define the bounded linear operator $\widetilde{N}$ on $b \mathscr{B}(E)$ as

$$
\widetilde{N} f(x)=\lambda_{o} \int_{E}[f(y)-f(x)] N_{x}^{\prime}(\mathrm{d} y) \text { for all } f \in b \mathscr{B}(E) \text { and } x \in E .
$$

Then $\widetilde{N}=N-\lambda I=\lambda_{o}\left(N^{\prime}-I\right)$ and it is the generator of a $C_{0}-$ semigroup $\left(P_{t}\right)_{t \geqslant 0}$ on $b \mathscr{B}(E)$,

$$
P_{t}:=\mathrm{e}^{t \widetilde{N}}, t \geqslant 0 .
$$

Each $P_{t}$ is a Markovian kernel on $E$, more precisely, $P_{t} f=\mathrm{e}^{-t \lambda_{o}} \sum_{k \geqslant 0} \frac{\left(\lambda_{o} t\right)^{k}}{k !} N^{\prime k} f$, where $N^{\prime k}:=\underbrace{N^{\prime} \circ \ldots \circ N^{\prime}}_{k \text { times }}$. The operator $\widetilde{N}$ is the generator of a (continuous time) pure jump Markov process $X=\left(X_{t}\right)_{t \geqslant 0}$ with state space $E$. Clearly, $\widetilde{N}$ is the weak generator of $\left(P_{t}\right)_{t \geqslant 0}$, with $\mathscr{D}(\widetilde{N})=b \mathscr{B}(E)$.

The scaling property. Assume that $E$ is a star-convex subset of $\mathbb{R}^{d}, d \geqslant 1$, i.e. there exists an $x_{o}$ in $E$ such that for all $x$ in $E$ the line segment from $x_{o}$ to $x$ is in $E$. For simplicity we suppose that $x_{o}=0$. For a real-valued function $f$ on $E$ and $s \in(0,1)$ we denote be $f_{s}$ the function on $E$ defined as $f_{s}(x):=f(s x), x \in E$.

Let $n \in \mathbb{Z}$. A linear operator $(L, \mathscr{D}(L))$ on $b \mathscr{B}(E)$ is called homogeneous of degree $n$ provided that for every $s \in(0,1)$ and $f \in \mathscr{D}(L)$ one has $f_{s} \in \mathscr{D}(L)$ and $(L f)_{s}=s^{n} L\left(f_{s}\right)$.

Clearly, the Laplace operator (in a star-convex subset of $\mathbb{R}^{d}$ ) is homogeneous of degree -2 . In the next section we shall give examples of operators related to fragmentation processes, satisfying such a scaling property.

We can state now the main result of this section.

Theorem 1. Let $n \in \mathbb{Z},\left(P_{t}\right)_{t \geqslant 0}$ be the transition function of a right (Markov) process $\left(X, \mathbb{P}^{x}\right)$ with state space $E$, let $(L, \mathscr{D}(L))$ be the weak generator of $\left(P_{t}\right)_{t \geqslant 0}$, and $\left(U_{\alpha}\right)_{\alpha>0}$ the associated resolvent. 
(i) The following assertions are equivalent.

(i.a) The transition function $\left(P_{t}\right)_{t \geqslant 0}$ satisfies

$$
\left(P_{t} f\right)_{s}=P_{t s^{n}}\left(f_{s}\right) \text { for all } f \in b p \mathscr{B}(E), s \in(0,1) \text {, and } t \geqslant 0 \text {. }
$$

(i.b) The resolvent family $\left(U_{\alpha}\right)_{\alpha>0}$ satisfies

$$
s^{n}\left(U_{\alpha} f\right)_{s}=U_{\frac{\alpha}{s^{n}}}\left(f_{s}\right) \text { for all } f \in b p \mathscr{B}(E), s \in(0,1), \text { and } \alpha>0
$$

(i.c) The weak generator $(L, \mathscr{D}(L))$ is homogeneous of degree $n$.

(i.d) The process $X$ has the following scaling property:

$$
\mathbb{E}^{s x}\left(X_{t} \in A\right)=\mathbb{E}^{x}\left(X_{t s^{n}} \in \frac{1}{S} A\right) \text { for all } A \in \mathscr{B}(E), x \in E, s \in(0,1) \text {, and } t \geqslant 0 .
$$

(ii) Assume that $N$ is a kernel on $E$ which is homogeneous of degree $n$. Then the pure jump Markov process having the generator $\widetilde{N}$ has the scaling property (3).

Proof. We clearly have $(i . a) \Longleftrightarrow(i . d)$ because $\left(P_{t}\right)_{t \geqslant 0}$ is the transition function of $X$.

$(i . a) \Longrightarrow(i . b)$. We have $\left(U_{\alpha} f\right)_{s}=\int_{0}^{\infty} e^{-\alpha t}\left(P_{t} f\right)_{s} \mathrm{~d} t=\int_{0}^{\infty} e^{-\alpha t} P_{t s^{n}}\left(f_{s}\right) \mathrm{d} t=$ $\frac{1}{s^{n}} U_{\frac{\alpha}{\rho^{n}}}\left(f_{s}\right)$, where we used the hypothesis $(i . a)$ to get the second equality.

$(i . b) \Longrightarrow(i . a)$. With the same computation as before, we get from $(i . b)$ that for all $\alpha>0$ we have $\int_{0}^{\infty} e^{-\alpha t}\left(P_{t} f\right)_{s} \mathrm{~d} t=\int_{0}^{\infty} e^{-\alpha t} P_{t s^{n}}\left(f_{s}\right) \mathrm{d} t$. Since any bounded $\beta$-level excessive function belongs to $\mathscr{B}_{o}$, using a monotone class argument, in order to prove (1) we may assume that $f \in \mathscr{B}_{o}$ and therefore the real-valued functions $t \longmapsto$ $\left(P_{t} f\right)_{s}(x)$ and $t \longmapsto P_{t s^{n}}\left(f_{s}\right)(x)$ are both right continuous on $[0, \infty)$ for every $x \in$ $E$. By the uniqueness property of the Laplace transform we conclude now that (1) holds.

(i.a) $\Longrightarrow($ i.c $)$. Let $f \in \mathscr{D}(L)$. Observe first that if $g \in \mathscr{B}_{0}$ then for all $s \in$ $(0,1)$ we have $g_{s} \in \mathscr{B}_{0}$ because $\lim _{t \backslash 0} P_{t}\left(g_{s}\right)=\lim _{t \searrow^{0}} P_{t s^{n}} g=g$. Consequently, if $f \in \mathscr{D}(L)$ then $(L f)_{s} \in \mathscr{B}_{0}$ and we have pointwise $(L f)_{s}=\lim _{t} \searrow_{0} \frac{\left(P_{t} f\right)_{s}-f_{s}}{t}=$ $s^{n} \lim _{t \backslash 0} \frac{P_{t s^{n}}\left(f_{s}\right)-f_{s}}{t s^{n}}=s^{n} L\left(f_{s}\right)$. As a consequence $f_{s}$ also belongs to $\mathscr{D}(L)$, hence $(L, \mathscr{D}(L))$ is homogeneous of degree $n$.

(i.c) $\Longrightarrow\left(\right.$ i.b). As before, in order to prove (2), we may suppose that $f \in \mathscr{B}_{0}$ and let $g:=U_{\alpha} f \in \mathscr{D}(L)$. Because $g_{s}$ also belongs to $\mathscr{D}(L)$, there exists $h \in \mathscr{B}_{O}$ such that $g_{s}=U_{\frac{\alpha}{s^{n}}} h$. We have $(L g)_{s}=\alpha\left(U_{\alpha} f\right)_{s}-f_{s}=\alpha g_{s}-f_{s}=\alpha U_{\frac{\alpha}{s^{n}}} h-f_{s}$. We have also $L\left(g_{s}\right)=L\left(U_{\frac{\alpha}{s^{n}}} h\right)=\frac{\alpha}{s^{n}} U_{\frac{\sigma^{n}}{n}} h-h$. The equality $(L g)_{s}=s^{n} L\left(g_{s}\right)$ is therefore equivalent with $\alpha U_{\frac{\alpha}{s^{n}}} h-f_{s}=\alpha U_{\frac{\alpha}{s^{n}}} h-s^{n} h$. Hence $f_{s}=s^{n} h, U_{\frac{\alpha}{s^{n}}}\left(f_{s}\right)=s^{n} U_{\frac{\alpha}{s^{n}}} h=$ $s^{n} g_{s}=s^{n}\left(U_{\alpha} f\right)_{s}$.

(ii) Observe first that the hypothesis on $N$ implies that $\lambda(s x)=(N 1)_{s}(x)=$ $s^{n} N 1(x)=s^{n} \lambda(x)$ for all $x \in E$ and $s \in(0,1]$. It follows that the kernel $\lambda I$ is homogeneous of degree $n$ and we deduce that $\widetilde{N}=N-\lambda I$ is also homogeneous of degree $n$. The scaling property of $X$ is now a consequence of the equivalence $(i . c) \Longleftrightarrow(i . d)$, since $\widetilde{N}$ is its generator. 
Remark 1. By Theorem 1 if follows that:

If $N$ is a kernel on $E$ which is homogeneous of degree $n$, then the induced semigroup $P_{t}=e^{t \widetilde{N}}, t \geqslant 0$, satisfies the scaling property (1).

One can give a direct, alternative proof for this assertion. Indeed, observe first that the hypothesis on $N$ implies that $\lambda(s x)=(N 1)_{s}(x)=s^{n} N 1(x)=s^{n} \lambda(x)$ for all $x \in E$ and $s \in(0,1]$. It results that the kernel $\lambda I$ is homogeneous of degree $n$ and we deduce that $\widetilde{N}=N-\lambda I$ is also homogeneous of degree $n$. Therefore $\left(\widetilde{N}^{k} f\right)_{s}=s^{k n} \widetilde{N}^{k}\left(f_{s}\right)$ for any $k \in \mathbb{N}^{*}$ and we conclude that $\left(P_{t} f\right)_{s}=\sum_{k \geqslant 0} \frac{t^{k}}{k !}\left(\widetilde{N}^{k} f\right)_{s}=\sum_{k \geqslant 0} \frac{\left(t s^{n}\right)^{k}}{k !} \widetilde{N}^{k}\left(f_{s}\right)=$ $P_{t s^{n}}\left(f_{s}\right)$.

\section{Scaling property for the SDE of fragmentation}

In this section we consider the framework from [2].

Discontinuous fragmentation kernels for avalanches. We describe first a binary fragmentation model. Consider an infinite system of particles, each particle being characterized by its mass. As time evolves the particles perform fragmentation, that is one particle can split into two smaller particles by conserving the total mass. Let $F$ be a fragmentation kernel, that is, a symmetric function $F:(0,1]^{2} \longrightarrow \mathbb{R}_{+}$. Here $F(x, y)$ represents the rate of fragmentation of a particle of size $x+y$ into two particles of sizes $x$ and $y$.

The following assumption is suggested by the so called rupture properties, emphasized in the deterministic modelling of the snow avalanches:

(H) There exists a function $\Phi:(0, \infty) \longrightarrow(0, \infty)$ such that $F(x, y)=\Phi\left(\frac{x}{y}\right)$ for all $x, y>0$.

Since the fragmentation kernel $F$ is assumed to be a symmetric function, we have $\Phi(z)=\Phi\left(\frac{1}{z}\right)$ for all $z>0$. An example is as follows. Fix a "ratio" $r, 0<r<1$, and consider the fragmentation kernel $F^{r}:[0,1]^{2} \longrightarrow \mathbb{R}_{+}$, defined as $F^{r}(x, y):=$ $\frac{1}{2}\left(\delta_{r}\left(\frac{x}{y}\right)+\delta_{1 / r}\left(\frac{x}{y}\right)\right)$, if $x, y>0$, and $F^{r}(x, y):=0$ if $x y=0$.

One can see that the fragmentation kernel $F^{r}$ satisfies condition $(H)$, more precisely we have $F^{r}(x, y)=\Phi^{r}\left(\frac{x}{y}\right)$ for all $x, y>0$, where $\Phi^{r}:(0, \infty) \longrightarrow(0, \infty)$ is defined as $\Phi^{r}(z):=\frac{1}{2}\left(\delta_{r}(z)+\delta_{1 / r}(z)\right), z>0$. Clearly, the function $\Phi^{r}$ is not continuous. By approximating the function $\Phi^{r}$ with a convenient sequence of continuous functions, one can see that the kernel $N^{F^{r}}$ associated with $F^{r}$ is given by the following linear combination of Dirac measures:

$$
N_{x}^{F^{r}}:=\lambda_{o}\left(\beta x \delta_{\beta x}+(1-\beta) x \delta_{(1-\beta) x}\right),
$$

where $\lambda_{o}:=\frac{\beta^{2}+(1-\beta)^{2}}{4}$ with $\beta:=\frac{r}{1+r}$. In this case the kernel $N^{F^{r}}$ is no more Markovian and has no density with respect to the Lebesgue measure. 
The corresponding stochastic differential equation of fragmentation. To emphasize the stochastic differential equation of fragmentation which is related to our stochastic model for the avalanches, we consider the kernel $N^{F^{r}}$ on $E:=[0,1]$ and the associated pure jump process $X=\left(X_{t}\right)_{t \geqslant 0}$ with state space $E$.

We state now the stochastic differential equation of fragmentation for avalanches:

$$
X_{t}=X_{0}-\int_{0}^{t} \int_{0}^{1}\left((1-\beta) X_{\alpha-} \mathbb{1}_{\left[\frac{s}{\beta \lambda_{o}}<X_{\alpha-} \leqslant 1\right]}+\beta X_{\alpha-} \mathbb{1}_{\left[\frac{s}{\lambda_{o}}<X_{\alpha-} \leqslant \frac{s}{\beta \lambda_{o}}\right]}\right) p(\mathrm{~d} \alpha, \mathrm{d} s),
$$

where $p(\mathrm{~d} \alpha, \mathrm{d} s)$ is a Poisson measure with intensity $q:=\mathrm{d} \alpha \mathrm{d} s$.

Recall that the solution $X$ of (5) describes the time evolution of the size of a typical particle as follows. At some exponential random instants of parameter $\lambda_{0}$, either, with probability $1-X$, no fragmentation occurs for the typical particle, or else, it breaks into two smaller particles: we subtract $(1-\beta) X$ from $X$ with probability $\beta X$, or $\beta X$ with probability $(1-\beta) X$. The conditions on the particle size are induced by the specific property of an avalanche, depending on $\beta$.

The existence of the weak solution to the equation (5) was proved in [2]. The next corollary shows that this solution satisfies the claimed scaling property.

Corollary 1. The weak solution of the stochastic differential equation of fragmentation for avalanches (5), with the initial distribution $\delta_{x}, x \in E$, is equal in distribution with $\left(X, \mathbb{P}^{x}\right)$ and the following scaling property holds:

$$
\mathbb{E}^{s x}\left(X_{t} \in A\right)=\mathbb{E}^{x}\left(X_{t s} \in \frac{1}{s} A\right) \text { for every } x \in E, t \geqslant 0, A \in \mathscr{B}(E) \text {, and } s \in(0,1] .
$$

Proof. We show first that the kernel $N^{F^{r}}$ is homogeneous of degree one $(n=1)$. It is sufficient to show that a kernel $K$ of the form $K f(x):=x f(\beta x)$ has this property. We have indeed $K f(s x)=s x f(\beta s x)=s K\left(f_{s}\right)(x)$.

The scaling property follows now by assertion (ii) of Theorem 1 (see also Remark 1) because we know that the generator of $X$ is $\widehat{N^{F^{r}}}$.

The fractal property. We consider a sequence $\left(d_{n}\right)_{n} \geqslant 1$ such that $d_{1}<\beta \leqslant 1 / 2$ and $d_{n+1} / d_{n}<\beta$ for all $n \geqslant 1$. Let $n \geqslant 1$ be fixed and define

$$
E_{n}:=\left[d_{n}, 1\right], E_{n}^{\prime}:=\left[d_{n+1}, d_{n}\right) \text {, and } E_{0}^{\prime}=E_{1} .
$$

Then clearly $E_{n}=\bigcup_{k=1}^{n} E_{k-1}^{\prime}$.

The kernel $N^{F^{r}}$ given by (4) is used to define the kernel $N_{n}^{r}$ on $E_{n}$ as

$$
N_{n}^{r} f:=\sum_{k=1}^{n} \mathbb{1}_{E_{k-1}^{\prime}} N^{F^{r}}\left(f \mathbb{1}_{E_{k-1}^{\prime}}\right) \text { for all } f \in \operatorname{pb\mathscr {B}}\left(E_{n}\right)
$$

Further, we consider the first order integral operator $\mathscr{F}_{n}^{r}$,

$$
\mathscr{F}_{n}^{r} f(x):={\widetilde{N_{n}^{r}}}^{r}(x)=\int_{E_{n}}[f(y)-f(x)]\left(N_{n}^{r}\right)_{x}(\mathrm{~d} y) \text { for all } f \in p b \mathscr{B}\left(E_{n}\right) \text { and } x \in E_{n} .
$$


The operator $\mathscr{F}_{n}^{r}$ is the generator of a (continuous time) jump Markov process $X^{r, n}=\left(X_{t}^{r, n}\right)_{t \geqslant 0}$. Its transition function is $P_{t}^{r, n}:=e^{\mathscr{F}_{n}^{r} t}, t \geqslant 0$.

For every $x \in E$ let

$$
E_{\beta, x}:=\left\{\beta^{i}(1-\beta)^{j} x: i, j \in \mathbb{N}\right\} \cup\{0\} \text { and } E_{\beta, x, n}:=E_{\beta, x} \cap E_{n} \text { for } n \geqslant 1 .
$$

We can state now the fractal property of the process $X^{r, n}$, proved in [2].

Theorem 2. If $n \geqslant 1$ then the following assertions hold for the Markov process $X^{r, n}$ with state space $E_{n}$ and transition function $\left(P_{t}^{r, n}\right)_{t \geqslant 0}$.

(i) If $t \geqslant 0$ and $x \in E_{n}$ then $P_{t}^{r, n}\left(\mathbb{1}_{(x, 1]}\right)(x)=0$.

(ii) For every $\phi \in p b \mathscr{B}\left(E_{n}\right)$ and each probability $v$ on $E_{n}$, the process $\phi\left(X_{t}^{r, n}\right)-\int_{0}^{t} \mathscr{F}_{n}^{r} \phi\left(X_{s}^{r, n}\right) \mathrm{d} s, t \geqslant 0$, is a martingale under $\mathbb{P}^{v}$, with respect to the natural filtration of $X^{r, n}$.

(iii) If $x \in E_{n}$ then $\mathbb{P}^{x}$-a.s. $X_{t}^{r, n} \in E_{\beta, x, n}$ for all $t \geqslant 0$.

\section{Numerical results}

Let $A \subset[0,1]$ be a fixed set and $x \in[0,1]$. By Corollary 1 for all $n \in \mathbb{N}^{*}$ and all time $t>0$ we have the following scaling property:

$$
\mathbb{E}^{x}\left(X_{t} \in A\right)=\mathbb{E}^{\frac{1}{n} x}\left(X_{n t} \in \frac{1}{n} A\right) \text { for all } t \geqslant 0 .
$$

The relation (6) indicates that the probability that the process starting from $x$ is in the set $A$ at time $t$ is exactly the probability that the process starting from $x / n$, belongs to the smaller set $A / n$ at time $n t$. The key point of the equality (6) is that it depends on $n$ only on the right hand side.

To test numerically the relation (6) we use a Monte Carlo simulation for the stochastic differential equation of fragmentation given by (5).

We fix a set $A \subset[0,1]$, a point $x \in[0,1]$, a final time $T \in \mathbb{R}_{+}^{*}$ and $n \in \mathbb{N}^{*}$. In the first step, we sample values of $X_{T}$ starting from $x$ as a solution of the corresponding stochastic differential equation of fragmentation with the discontinuous kernel $F^{r}$, by using the algorithm developed and implemented in [3]. For the reader's convenience we recall it below, we can remark the fractal property of the resulting fragments after the splitting, property which holds according to assertion (iii) of Theorem 2. In the second step we compute the probability that the samples of $X_{T}$ belong to the set $A$. Then, we compare it with the probability that the process $X_{n T}$, starting from $x / n$, belongs to $\frac{1}{n} A$.

We fix the parameter $\beta<\frac{1}{2}$ and a final time $T$.

\section{Algorithm}


Step 0: Sampling the initial particle $X_{0} \sim Q_{0}$

Step p: Sampling a random variable $S_{p} \sim \operatorname{Exp}\left(\lambda_{0}\right)$

$$
\begin{aligned}
& \text { Set } T_{p}=T_{p-1}+S_{p} \\
& \text { Set } X_{t}=X_{p-1} \text { for each } t \in\left[T_{p-1}, T_{p}\right)
\end{aligned}
$$$$
\text { Set } X_{p}=\beta X_{p-1} \text { with probability } \beta X_{p-1} \text {, }
$$

Stop: When $T_{p}>T$.

$$
\begin{aligned}
& X_{p}=(1-\beta) X_{p-1} \text { with probability }(1-\beta) X_{p-1}, \\
& \text { or } X_{p}=X_{p-1} \text { with probability } 1-X_{p-1}
\end{aligned}
$$

Outcome: The approximated particle mass at time $T, X_{p-1}$.

To implement the above Monte Carlo simulation associated to the relation (6), we fix the set $A$ a union of disjoint intervals, $A=\left[0, \frac{1}{4}\right] \cup\left[\frac{1}{2}, \frac{3}{4}\right]$, the starting point $x=1$, that does not belong to $A$. We consider the Monte Carlo parameter $10^{4}$. Notice that the fractal character of the particles is encoding in the ratio $\beta$.

In Table 1 below we give the Monte Carlo estimator for each one of the terms of relation (6), for $\beta=\frac{1}{6}, T=20$ fixed, and different values of $n$. In Table 2 we illustrate the Monte Carlo estimator of the each term of relation (6) for $n=3, T=20$ fixed, and different values of $\beta$. In Table 3 is written down the Monte Carlo estimator of the each term of relation (6), for different values of $n$, with parameter $10^{4}$.

Table 1 Monte Carlo estimators for $10^{4}$ simulations for $x=1, \beta=\frac{1}{6}, T=20$ fixed, and different values of $n$

\begin{tabular}{lll}
\hline $\mathrm{n}$ & $\mathbb{E}^{x}\left(X_{t} \in A\right)$ & $\mathbb{E}^{\frac{1}{n} x}\left(X_{t} \in \frac{1}{n} A\right)$ \\
\hline 3 & 1 & 0.9998 \\
10 & 1 & 1 \\
20 & 1 & 1 \\
30 & 1 & 1 \\
40 & 1 & 1 \\
50 & 1 & 1 \\
\hline
\end{tabular}

Table 2 Monte Carlo estimators for $10^{4}$ simulations for $x=1, n=3, T=20$ fixed, and different values of $\beta$

\begin{tabular}{lll}
\hline $\mathrm{n}$ & $\mathbb{E}^{x}\left(X_{t} \in A\right)$ & $\mathbb{E}^{\frac{1}{n} x}\left(X_{t} \in \frac{1}{n} A\right)$ \\
\hline$\frac{1}{6}$ & 1 & 0.9998 \\
$\frac{1}{3}$ & 1 & 1 \\
$\frac{1}{9}$ & 1 & 1 \\
\hline
\end{tabular}

We represent in Figure 1 the evolution in time of $t \longmapsto \mathbb{E}^{x}\left(X_{t} \in A\right)$ the red color and $t \longmapsto \mathbb{E}^{\frac{1}{n} x}\left(X_{n t} \in \frac{1}{n} A\right)$ the blue color in the time interval $t \in[50,100]$ for $\beta, A, x$ 
Table 3 Monte Carlo estimators for $10^{4}$ simulations for $x=1, n=3, \beta=\frac{1}{6}$ fixed, and different values of $T$

\begin{tabular}{lll}
\hline $\mathrm{n}$ & $\mathbb{E}^{x}\left(X_{t} \in A\right)$ & $\mathbb{E}^{\frac{1}{n} x}\left(X_{t} \in \frac{1}{n} A\right)$ \\
\hline 20 & 1 & 1 \\
\hline 30 & 1 & 1 \\
\hline
\end{tabular}

Fig. 1 The path of Monte Carlo approximation for $t \longmapsto$ $\mathbb{E}^{x}\left(X_{t} \in A\right)$, the red color, and $t \longmapsto \mathbb{E}^{\frac{1}{n} x}\left(X_{n t} \in \frac{1}{n} A\right)$ the blue color, for $\beta=\frac{1}{6}$, $A=\left[0, \frac{1}{4}\right] \cup\left[\frac{1}{2}, \frac{3}{4}\right], n=3$, the Monte Carlo parameter $10^{4}$, and the Euler step $10^{-3}$.

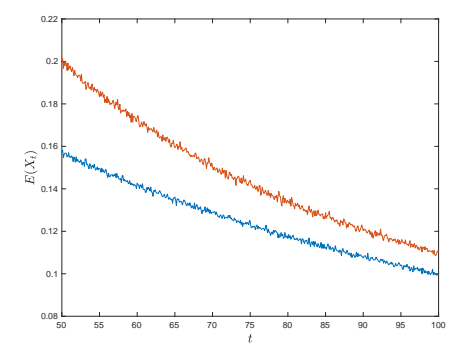

chosen above, and $n=3$. Remark that in large time the red trajectory is very close to the blue one, that suggest the validity of the relation (6).

Acknowledgements For the third named author this work was supported by a grant of the Romanian National Authority for Scientific Research and Inovation, CNCS-UEFISCDI, project number PN-III-P1-1.1-PD- 2016-0293, within PNCDI III. Support from GDRI ECO-Math is kindly acknowledged.

\section{References}

1. Beznea, L., Deaconu, M., and Lupaşcu, O.: Branching processes for the fragmentation equation. Stoch. Proc. Appl. 25, 1861-1885 (2015)

2. Beznea, L., Deaconu, M., and Lupaşcu, O.: Stochastic equation of fragmentation and branching processes related to avalanches. J. Stat. Phys. 162, 824-841 (2016)

3. Beznea, L., Deaconu, M., and Lupaşcu-Stamate, O.: Numerical approach for stochastic differential equations of fragmentation; application to avalanches. Mathematics and Computers in Simulation. 160, 111-125 (2019)

4. Beznea, L., and Lupaşcu, O.: Measure-valued discrete branching Markov processes. Trans. Amer. Math. Soc. 368, 5153-5176 (2016) 
5. Calvez, V., Doumic, M., and Gabriel, P.: Self-similarity in a general aggregationfragmentation problem. Application to fitness analysis. J. Math. Pures Appl. 98, 1-27 (2012)

6. Dynkin, E.B.: Markov Processes. Vol. I, Springer (1965)

7. Escobedo, M., Mischler, S., and Rodriguez Ricard, M.: On self-similarity and stationary problem for fragmentation and coagulation models. Ann. I. H. Poincaré. 22, 9-125 (2005)

8. Ethier, N.S., and Kurtz, T.G.: Markov Processes-Characterization and Convergence. WilleyInterscience (1986)

9. Fitzsimmons, P.J.: Construction and regularity of measure-valued Markov branching processes. Israel J. Math. 64, 337-361 (1988)

10. Perthame B., and Ryzhik, L.: Exponential decay for the fragmentation or cell-division equation. J. Diff. Equations. 210, 155-177 (2005) 